\title{
Identificação dos usuários segundo nível de satisfação nos módulos gerontológicos empregando a análise de conglomerados
}

\author{
IDENTIFICATION OF USERS USING CLUSTER ANALYSIS ACCORDING TO DEGREE OF \\ SATISFACTION WITH GERIATRIC CARE SERVICES
}

\section{IDENTIFICACIÓN DE LOS PACIENTES SEGÚN NIVEL DE SATISFACCIÓN EN MÓDULOS GERONTOLÓGICOS EMPLEANDO EL ANÁLISIS DE CONGLOMERADOS}

\author{
Gustavo Nigenda-López ${ }^{1}$, Aarón Salinas-Rodríguez², Mariana Gómez-Camponovo³ ${ }^{3}$ Betty \\ Manrique-Espinoza ${ }^{4}$, José Arturo Ruiz-Larios ${ }^{5}$, Alejandra Trejo-Rosales ${ }^{6}$
}

\section{RESUMO}

Esta pesquisa teve como objetivos identificar os grupos de usuários segundo o grau de satisfação com a atenção recebida nos Módulos Gerontológicos e determinar os principais fatores associados. Trata-se de estudo transversal realizado com 181 usuários nos 36 módulos do Seguro Social dos Trabalhadores Públicos, no México. O nível de satisfação foi avaliado segundo três aspectos: características gerais da atenção recebida, amabilidade no trato e infraestrutura. Foi realizada uma análise de conglomerados para identificar grupos de usuários segundo o grau de satisfação e um modelo de regressão logística ordinal para identificar os fatores associados. Estiveram satisfeitos com o serviço $53 \%$ dos usuários; medianamente satisfeitos, $34,3 \%$; e insatisfeitos, $12,7 \%$. Os principais fatores associados com maior grau de satisfação foram: ser mulher, maior idade e ser chefe/a de família. O sistema de saúde deve dirigir sua atenção para esse grupo populacional que é crescente e incentivar o desenvolvimento de uma atenção de qualidade que contemple as suas necessidades.

\section{DESCRITORES}

Idoso

Satisfação do paciente

Serviços de Saúde para Idosos

México

\section{ABSTRACT}

The aim of this study was to identify groups of users according to their degree of satisfaction with geriatric care services and determine the primary factors associated with satisfaction. This was a cross-sectional study of 181 people enrolled in 36 modules pertaining to the State Workers Social Security Institute (ISSSTE) in Mexico. Degree of satisfaction was measured according to the following three areas: general characteristics of services offered, friendliness of staff and infrastructure. A cluster analysis was performed to identify groups of users according to their level of satisfaction, and an ordinal logistic regression model was used to determine the associated factors. Fifty-three percent were satisfied with the services, $34.3 \%$ were fairly satisfied and $12.7 \%$ were dissatisfied. The main characteristics associated with a greater degree of satisfaction were being female, older and the head of household. The health system must address this growing population and ensure the development of quality care to meet their needs.

\section{DESCRIPTORS \\ Aged \\ Patient satisfaction \\ Health Services for the Aged \\ Mexico}

\section{RESUMEN}

Se objetivó identificar grupos de usuarios según grado de satisfacción con la atención recibida en Módulos Gerontológicos, y determinar los principales factores asociados. Estudio transversal, muestra de 181 pacientes de los 36 módulos del Seguro Social de Trabajadores Estatales, en México. El nivel de satisfacción fue evaluado bajo tres aspectos: características generales de la atención recibida; amabilidad en el trato e infraestructura. Se realizó análisis de conglomerados para identificar grupos de pacientes según grado de satisfacción y un modelo de regresión logística ordinal para identificar factores asociados. Resultaron satisfechos con el servicio $53 \%$ de los pacientes; medianamente satisfechos $34,3 \%$ e insatisfechos $12,7 \%$. Los principales factores asociados a mayor grado de satisfacción fueron: ser mujer, mayor edad, ser jefe/a de familia. El sistema de salud debe dirigir su atención a este grupo poblacional creciente, e incentivar el desarrollo de una atención calificada que contemple sus necesidades.

\section{DESCRIPTORES}

Anciano

Satisfacción del paciente

Servicios de Salud para Ancianos México

\footnotetext{
${ }^{1}$ Doutorado em Política da Saúde. Pesquisador do Instituto Nacional de Saúde Pública do México e Pesquisador Associado do Harvard Global Equity Initiative da Faculdade de Medicina de Harvard. Boston, Estados Unidos da América. gustavo_nigenda@hms.harvard.edu ${ }^{2}$ Mestre em Ciências de Bioestatística. Pesquisador de Ciências da Saúde do Instituto de Saúde Pública do México. Cidade do México, México. asalinas@insp.mx ${ }^{3}$ Doutora em Ciências de Saúde Pública. Pesquisadora de Ciências da Saúde do Instituto de Saúde Pública do México e Professora Associada de Medicina Preventiva e Social da Faculdade de Medicina da Universidad de la República Uruguay. Montevideo, Uruguay. marianagomezc@higiene.edu.uy ${ }^{4}$ Doutor em Ciências de Epidemiologia. Pesquisador de Ciências da Saúde do Instituto de Saúde Pública do México. Cidade do México, México. bmanrique@insp.mx ${ }^{5}$ Sociólogo. Pesquisador de Ciências da Saúde do Instituto de Saúde Pública do México. Cidade do México, México. jose.ruiz@insp.mx ${ }^{6}$ Economista. Estudante Bolsista do Instituto de Saúde Pública do México. Cidade do México, México. ale.trejo04@gmail.com
} 


\section{INTRODUÇÃO}

A população idosa está em crescimento e continuará representando uma proporção maior da população mundial global nos próximos anos. Segundo estimativas das Nações Unidas, a população idosa global crescerá de 600 milhões para 2 bilhões dentro dos próximos 50 anos (entre 2000 e 2050). Este fenômeno também ocorrerá na América Latina e na região do Caribe, onde espera-se que a população idosa cresça de 42 milhões para 100 milhões entre 2000 e 2025 devido a mudanças substanciais e contínuas na estrutura etária da população. Esse processo de envelhecimento rápido da população apresenta desafios para vários países, incluindo o México, onde a população idosa tem apresentado um crescimento constante ao longo dos últimos 25 anos. Estimativas para 2010 indicam uma população idosa acima de 60 anos de mais de 10 milhões, representando $8,9 \%$ da população total. Esse fenômeno pressiona os sistemas previdenciários e aumenta a demanda por assistência médi$\mathrm{ca}^{(1)} \mathrm{e}$ a necessidade de trabalho qualificado em serviços geriátricos. De forma semelhante, ele conduzirá a uma demanda maior por assistência médica a longo prazo(2). Estudos publicados amplamente reconhecem que o conceito de qualidade é composto por pelo menos duas dimensões identificáveis e analisáveis-a técnica e a interpessoal. A primeira abrange processos administrativos, clínicos e técnicos envolvidos na produção do serviço; enquanto a última se refere à relação entre fornecedores e usuários do serviço, com foco na garantia de direitos fundamentais, tratamento justo, acesso à informação e satisfação com esses serviços. A distribuição de serviços de saúde adequados a idosos promove o envelhecimento ati$v o^{(3)}$, definido como o processo de otimizar oportunidades de saúde, participação ativa e segurança, as quais, juntas, incrementam a qualidade de vida à medida que as pessoas envelhecem. Instituições previdenciárias no México têm demonstrado preocupação à medida que segmentos progressivamente mais amplos de seus beneficiários atingem 60 ou 65 anos de idade. De todas as instituições públicas fornecendo serviços de saúde no país, o Instituto Mexicano de Serviços Previdenciários para Funcionários do Estado (Instituto de Seguridad y Servicios Sociales de los Trabajadores del Estado - ISSSTE) atende a população beneficiária mais idosa, razão pela qual lançou a iniciativa dos Módulos Gerontológicos (MG) em 1997, a fim de oferecer serviços de saúde individuais e com base neste grupo populacional. MG são espaços físicos localizados dentro das unidades de atenção de saúde primária do ISSSTE e especificamente equipados para fornecer atendimento ambulatorial a pessoas acima de 60 anos, encaminhadas com diagnóstico de enfermidade crônica. Os Módulos Gerontológicos são compostos por técnicos e profissionais que recebem treinamento básico em atenção à saúde deste grupo populacional. O principal objetivo desses módulos é fornecer atendimento contínuo aos seus usuários, inclusive mantendo indicadores biológicos dentro de limites razoáveis, prescrevendo e suprindo medicamentos, fornecendo informações sobre como controlar enfermidades em casa e organizando grupos de autoajuda para melhorar o gerenciamento da enfermidade através da interação social. A identificação e consideração das perspectivas dos usuários em relação ao desempenho dos serviços ${ }^{(4-5)}$ são estratégias para melhorar o atendimento de saúde e construir um sistema de saúde forte que conecte ativamente todas as partes envolvidas. Por esta razão, e devido às implicações de uma crescente população idosa, o objetivo deste estudo foi identificar grupos de usuários, através da análise de conglomerados, de acordo com seu grau de satisfação com os serviços oferecidos nos módulos de atendimento geriátrico e determinar fatores associados.

\section{MÉTODO}

\section{Desenho de estudo e população}

Foi utilizada uma amostra analítica composta por idosos participantes do estudo transversal A Satisfação dos Idosos com os Módulos Gerontológicos da Cidade do México e Fatores Associados, desenvolvido em Abril de 2010. Os 181 participantes foram selecionados com base em uma seleção de amostra não-probabilística de usuários idosos de 36 Módulos Gerontológicos do ISSSTE na Cidade do México. O estudo empregou um questionário estruturado que havia sido previamente avaliado quanto a sua compreensão durante uma fase piloto conduzida com sete usuários idosos de um MG.

Funcionários treinados pelo Instituto $\mathrm{Na}$ cional de Saúde Pública conduziram entrevistas pessoalmente com os idosos participantes em salas de espera de cada MG. Após a obtenção do Termo de Consentimento Livre e Informado, os idosos que concordaram em participar foram entrevistados, até que se atingisse a cota de cinco entrevistas por MG. Os entrevistadores coletaram informações referentes as características sócio-demográficas, socioeconômicas e relacionadas a saúde dos idosos. Os entrevistados também foram questionados quanto as condições do serviço do MG e aspectos gerais do atendimento recebido.

\section{Medição de variáveis e análise estatística}

A variável dependente - grau de satisfação dos idosos quanto à atenção recebida no MG, foi operacionalizada da seguinte forma:

Um indicador de satisfação de atenção percebida foi construído, utilizando oito variáveis para três áreas dife-
Identificação dos usuários segundo nível de satisfação nos módulos gerontológicos empregando a analise de conglomerados Nigenda-López G, Salinas-Rodríguez A, Gómez-Camponovo M, Manrique-Espinoza B, Ruiz-Larios JA, Trejo-Rosales A 
rentes: 1) características do módulo (limpeza, sinalização, acessibilidade física, oportunidade de consultas); 2) cordialidade dos funcionários (médicos, enfermeiros, administradores) e 3) infraestrutura. Foi considerada uma pontuação de 1 a 10 pontos para cada uma dessas variáveis (itens), sendo que o valor mais alto indicava maior satisfação. Uma nova variável, expressa como a simples soma total de todos os 18 itens, foi então construída para medir a satisfação global do usuário, com pontuações mais altas indicando maior satisfação. Baseado na suposição de que a população do estudo pudesse ser segmentada em grupos de acordo com seu grau de satisfação com o atendimento recebido, utilizou-se a análise de conglomerados (com os oito itens classificando satisfação) para identificar potenciais grupos de indivíduos em termos de qualidade percebida. Especificamente, utilizamos o método de partição $k$-means e um dendrograma para selecionar o número de grupos ${ }^{(6)}$.

Dado que os grupos definidos por análise de conglomerados podiam ser ordenados em uma escala de insatisfeito a satisfeito, utilizou-se um modelo de análise de regressão logística ordinal para identificar os fatores associados a satisfação com o cuidado recebido. Adicionalmente, uma vez que a percepção dos usuários de um mesmo MG poderia estar correlacionada, as análises estatísticas utilizaram estimadores robustos de variação para justificar uma falta de independência entre as observações ${ }^{(7)}$. Para a análise da relação entre o nível de satisfação e as variáveis independentes, utilizou-se o teste Kruskal-Wallis para variáveis contínuas e testes qui-quadrado para variáveis categóricas. Todas as análises estatísticas foram realizadas com uso do pacote estatístico STATA.

O Instituto Nacional de Ética de Saúde Pública (Cl: 513, № 239), Biossegurança e os Comitês de Pesquisa aprovaram o projeto original, e o Termo de Consentimento Livre e Informado foi obtido.

\section{RESULTADOS}

Baseado na pontuação obtida com a soma das oito variáveis compondo a avaliação de satisfação, a análise de conglomerados identificou três grupos potenciais de idosos quanto a satisfação percebida com o cuidado: $53 \%$ dos indivíduos foram classificados como satisfeitos, $34,3 \%$ como razoavelmente satisfeitos e $12,7 \%$ como insatisfeitos.

A Tabela 1 apresenta as características sócio-demográficas dos participantes, assim como as medidas de condição de saúde percebida. A amostra apresentou predominância de mulheres e indivíduos acima dos 70 anos, além de um baixo número total de profissionais. Há também proporções maiores de usuários que receberam atendimento no MG por mais de um ano, que reportaram ter recebido todos ou a maioria dos medicamentos e que percebem sua condição de saúde como boa ou regular. Os participantes apresentaram maior dificuldade com relação a mobilidade em comparação a outros tipos de limitações e $12,2 \%$ da amostra relataram morar sozinhos.

Tabela 1 - Características sócio-demográficas dos usuários do MG - Cidade do México, 2010

\begin{tabular}{|c|c|c|}
\hline Variável & $\mathbf{N}$ & $\%$ \\
\hline \multicolumn{3}{|l|}{ Sexo } \\
\hline Mulher & 125 & $69,1 \%$ \\
\hline Homem & 56 & $30,9 \%$ \\
\hline \multicolumn{3}{|l|}{ Idade } \\
\hline Média & 73,25 & \\
\hline Desvio padrão & 8,01 & \\
\hline \multicolumn{3}{|l|}{ Escolaridade completa } \\
\hline Nível profissional & 14 & $7,7 \%$ \\
\hline Ensino médio & 36 & $19,9 \%$ \\
\hline Ensino fundamental II & 28 & $15,5 \%$ \\
\hline Ensino fundamental I & 90 & $49,7 \%$ \\
\hline Nenhuma educação formal & 13 & $7,2 \%$ \\
\hline \multicolumn{3}{|l|}{ Arranjo de moradia } \\
\hline Com outra pessoa & 159 & $87,8 \%$ \\
\hline Sozinho & 22 & $12,2 \%$ \\
\hline \multicolumn{3}{|l|}{ Chefe de família } \\
\hline Idoso é o chefe de família & 93 & $51,4 \%$ \\
\hline Idoso não é o chefe de família & 88 & $48,6 \%$ \\
\hline \multicolumn{3}{|l|}{ Cuidador } \\
\hline Idoso tem um cuidador & 114 & $63 \%$ \\
\hline Idoso não tem um cuidador & 67 & $37 \%$ \\
\hline \multicolumn{3}{|l|}{ Medicamentos recebidos } \\
\hline Todos/maioria & 139 & $76,8 \%$ \\
\hline Alguns/poucos/nenhum & 42 & $23,2 \%$ \\
\hline \multicolumn{3}{|l|}{ Tempo utilizando o módulo } \\
\hline$<1$ ano & 50 & $27,6 \%$ \\
\hline 1 a 5 anos & 92 & $50,8 \%$ \\
\hline 5 anos ou mais & 39 & $21,6 \%$ \\
\hline \multicolumn{3}{|l|}{ Condição de saúde percebida } \\
\hline Muito boa & 10 & $5,5 \%$ \\
\hline Boa & 73 & $40,3 \%$ \\
\hline Regular & 75 & $41,4 \%$ \\
\hline Ruim & 21 & $11,6 \%$ \\
\hline Muito ruim & 2 & $1,1 \%$ \\
\hline \multicolumn{3}{|l|}{ Dificuldade reportada com } \\
\hline Sem dificuldades físicas & 80 & $44,2 \%$ \\
\hline Mobilidade & 69 & $38,1 \%$ \\
\hline Audição & 20 & $11,1 \%$ \\
\hline Braços/mãos & 6 & $3,3 \%$ \\
\hline Visão & 5 & $2,8 \%$ \\
\hline Não conhecida & 1 & $0,5 \%$ \\
\hline
\end{tabular}

A Tabela 2 apresenta os resultados de uma análise comparativa das características dos idosos de acordo com seu nível de satisfação com o atendimento. MuIheres ( $p<0,001)$, indivíduos mais velhos $(p<0,006)$ e aqueles que receberem atendimento no MG por um período maior de tempo $(p<0,035)$ expressaram maior satisfação, enquanto pessoas que se consideravam em condição de saúde ruim ou muito ruim estavam menos satisfeitas $(p<0,000)$. 
Tabela 2 - Características da amostra por nível de satisfação - Cidade do México, 2010

\begin{tabular}{|c|c|c|c|c|c|}
\hline \multirow[t]{2}{*}{ Co-variável } & & Satisfeito & $\begin{array}{c}\text { Razoavelmente } \\
\text { satisfeito }\end{array}$ & Insatisfeito & \multirow[t]{2}{*}{ Valor-p* } \\
\hline & & $\mathrm{n}=96$ & $\mathrm{n}=62$ & $\mathrm{n}=23$ & \\
\hline Mulher & $\%$ & $74,0 \%$ & $74,2 \%$ & $34,8 \%$ & 0,001 \\
\hline Idade (anos) & Média & $\begin{array}{l}74,48 \\
(0,84)\end{array}$ & $\begin{array}{l}73,02 \\
(0,98)\end{array}$ & $\begin{array}{l}68,78 \\
(1,25)\end{array}$ & 0,006 \\
\hline Idoso é chefe de família & $\%$ & $56,3 \%$ & $45,2 \%$ & $47,8 \%$ & 0,370 \\
\hline Idoso não tem um cuidador & $\%$ & $43,8 \%$ & $37,1 \%$ & $8,7 \%$ & 0,008 \\
\hline $\begin{array}{l}\text { Condição de saúde auto-reportada do } \\
\text { idoso - muito ruim/ ruim/ regular }\end{array}$ & $\%$ & $40,6 \%$ & $64,5 \%$ & $82,6 \%$ & $<0,000$ \\
\hline Tempo utilizando o módulo (dias) & Média & $\begin{array}{l}1071,68 \\
(82,88) \\
\end{array}$ & $\begin{array}{l}797,47 \\
(91,93) \\
\end{array}$ & $\begin{array}{c}672,52 \\
(113,07) \\
\end{array}$ & 0,035 \\
\hline
\end{tabular}

* valor-p para o teste qui-quadrado ou Kruskall-Wallis.

( ): Desvio padrão.

Os fatores associados a satisfação percebida serão demonstrados na Tabela 3. Conforme indicado pelas estatísticas descritivas acima, as variáveis observadas como fortemente associadas a maiores níveis de satisfação são relacionadas a indivíduos do sexo feminino e com idade mais avançada. As variáveis de condições de vida que aumentam a probabilidade de satisfação são: ser o chefe de família, não ter um cuidador e ter recebido atendimento geriátrico no MG por um período maior de tempo. Por outro lado, uma percepção ruim da condição de saúde geral diminui a probabilidade de que o participante se sinta satisfeito com a atenção recebida.

Tabela 3 - Fatores associados a satisfação dos idosos - Cidade do México, 2010

\begin{tabular}{lcccc}
\hline Variável & OR & $\begin{array}{c}\text { Erro } \\
\text { padrão* }\end{array}$ & \multicolumn{2}{c}{ IC $_{95 \%}$} \\
\hline Mulher & 2,8631 & 1,1890 & 1,2686 & 6,4615 \\
Idade (anos) & 1,0751 & 0,0245 & 1,0281 & 1,1242 \\
Idoso é chefe de família & 2,4757 & 0,8789 & 1,2346 & 4,9647 \\
$\begin{array}{l}\text { Idoso não tem um } \\
\text { cuidador }\end{array}$ & 2,5135 & 0,8948 & 1,2510 & 5,0502 \\
$\begin{array}{l}\text { Condição de saúde } \\
\text { auto-reportada do idoso - } \\
\text { muito ruim/ ruim/ regular }\end{array}$ & 0,2476 & 0,0976 & 0,1143 & 0,5363 \\
$\begin{array}{l}\text { Tempo utilizando } \\
\text { o módulo (dias) }\end{array}$ & 1,0007 & 0,0002 & 1,0002 & 1,0011 \\
$\begin{array}{l}\text { Intercepção 1 } \\
\text { Intercepção 2 }\end{array}$ & 4,2871 & 1,9500 & & \\
\end{tabular}

* Erros padrões robustos, considerando o grau de correlação dos idosos dentro do módulo gerontológico.

\section{DISCUSSÃO}

Ao contrário de um estudo no qual o sexo não estava associado com a satisfação, os indivíduos participantes do presente estudo que estavam satisfeitos ou razoavelmente satisfeitos eram predominantemente do sexo femini$\mathrm{no}^{(8)}$. Entretanto, várias características associadas a uma maior satisfação são consistentes com os resultados de estudos anteriores, incluindo ter uma idade mais avança$\mathrm{da}^{(9-12)}$, ser o chefe de família, não tem um cuidador, ser um usuário antigo do MG e considerar sua condição de saúde como boa ou muito boa ${ }^{(8,10,12)}$. Além disso, mulheres com um maior grau de autonomia(13) podem se beneficiar mais desta atenção focada. O perfil do grupo de usuários satisfeitos contrasta com aquele do grupo de insatisfeitos, sendo que este último tende a ser composto por indivíduos do sexo masculino, mais jovens, que tem um cuidador, percebem sua condição de saúde como ruim ou muito ruim e utilizam os serviços do MG há menos tempo; resultados que coincidem com outro estudo ${ }^{(10)}$.

Diferentemente de estudos que contaram com uma análise de dados pré-existentes ${ }^{(8,10,13)}$, este estudo permitiu a exploração de questões que não haviam sido reportadas anteriormente por outros autores, principalmente devido ao método utilizado para obter informações diretamente de usuários de atenção primária no local efetivo em que o cuidado foi recebido. Apesar de outras pesquisas coletarem informações sobre os serviços ${ }^{(14-17)}$, elas não se dirigiram especificamente aos idosos como um grupo populacional, e a maioria se baseou em inquéritos com características distintas. As variáveis compondo a pontuação para determinar nossa medida de satisfação tratam de uma variedade de aspectos do suprimento de atenção que foram empregados por vários estudos em países diferentes. Estas variáveis incluem tratamento interpessoal ${ }^{(16)}$ acessibilidade $^{(15)}$ infraestrutura $^{(16)}$ e processos de cuidado. A partir de uma perspectiva econômica, o aumento de custos associados com o envelhecimento da população não é necessariamente resultante da idade avançada em si, mas do número elevado de enfermidades crônicas que acompanham o fenômeno ${ }^{(1,13)}$. Portanto, o desenvolvimento de políticas que promovam a qualidade de vida em uma idade mais avançada ${ }^{(17-18)}$ proporcionará resultados mais efetivos e duradouros.

Este estudo tem limitações inerentes a análises transversais. Primeiramente, é difícil identificar se a atenção fornecida pelos módulos melhorou ao longo do tempo. Adicionalmente, uma vez que os participantes do estudo eram parte de uma amostra de conveniência, em vez de uma amostra representativa, as pessoas satisfeitas podem ter sido sobre-representadas. Ademais, as entrevistas foram conduzidas enquanto os participantes ainda estavam no módulo, o que pode ter influenciado suas respostas; entretanto, para evitar o viés da avaliação, os entrevistadores não pertenciam ao ISSSTE e eram treinados nessa questão. Outra limitação inerente a este tipo de pesquisa é sua alta dependência das características sociais e expectativas dos usuários ${ }^{(19)}$. Apesar das limitações mencionadas acima, o estudo explora fenômenos que não foram estudados inteiramente até o
Identificação dos usuários segundo nível de satisfação nos módulos gerontológicos empregando a analise de conglomerados Nigenda-López G, Salinas-Rodríguez A, Gómez-Camponovo M, Manrique-Espinoza B, Ruiz-Larios JA, Trejo-Rosales A 
momento. Um aumento na demanda de populações idosas como consequência do aumento das enfermidades crônicas exigirá uma resposta das instituições de saúde dos países em desenvolvimento, envolvendo ações de atenção primária, o que está se tornando cada vez mais urgente.

\section{CONCLUSÃO}

O presente estudo apresentou uma ferramenta potencialmente útil para avaliar a satisfação e uma técnica de análise inovadora. Este é especialmente o caso considerando que estudos de pesquisa insistem em utilizar este tipo de medição afim de aprimorar o desempenho de gerenciamento. Sendo assim, as percepções dos usuários quanto à satisfação com a atenção recebida é extremamente importante para a qualidade, uma vez que uma melhor atenção conduz a melhor presença e adesão ao tratamento. Estas condições possibilitam o progresso em direção a melhorar a qualidade de vida nos últimos estágios do ciclo da vida.

\section{REFERÊNCIAS}

1. Ham Chande R. Enfoques y perspectivas sobre el envejecimiento en Mexico. In: Salgado N, Wong R, editores. Envejeciendo en la pobreza: género, salud y calidad de vida. Mexico: Edamsa; 2003. p. 81-96.

2. Gibson DA, Moorin RE, Pree DB, Emery JD, Holman CD. Effects of the Medicare enhanced primary care program on primary care physician contact in the population of older Western Australians with chronic diseases. Aust Health Rev. 2011;35(3):334-40.

3. Mendoza Nuńez VM. Aspectos psicosociales de las enfermedades crónicas en la vejez en el contexto de pobreza. In: Salgado N, Wong R, editores. Envejeciendo en la pobreza: género, salud y calidad de vida. Mexico: Edamsa; 2003. p. 57-80.

4. Donabedian A. Epidemiologia de la calidad. Rev Calid Asist. 2001;16(1):54-62.

5. Gaioso VP, Mishima SM. User satisfaction from the perspective of acceptability in the family health scenario. Texto Contexto Enferm. 2007;16(4):617-25.

6. Everitt BS, Landau S, Leese M, Srhal D. Cluster analysis. 2th ed. London: John Wiley \& Sons; 2000.

7. Rogers WH. Regression standard errors in clustered samples. Stata Techn Bull. 1994;3(13):19-23.

8. Sauceda-Valenzuela AL, Wirtz V, Santa-Ana Téllez Y, Kageyama-Escobar ML. Ambulatory health services users experience of waiting time and expenditure and factors associated with perception of low quality of care in Mexico. BMC Health Serv Res. 2010;10:178.

9. Cruz WBS, Melleiro MM. Assessment levels of the user's satisfaction in a private hospital. Rev Esc Enferm USP [Internet]. 2010 [cited 2011 Jan 23];44:147-52. Available from: http:// www.scielo.br/pdf/reeusp/v44n1/en_a21v44n1.pdf

10. Puig A, Pagin JA, Wong R. Assessing quality across healthcare subsystems in Mexico. J Ambul Care Manage. 2009;87(2):271-8.
11. Bleich SN, Ozaltin E, Murray CK. How does satisfaction with the health-care system relate to patient experience? Bull World Health Organ. 2009;87(4):271-8.

12. Xiao H, Barber J. The effect of perceived health status on patient satisfaction. Value Health. 2008;11(4):719-25.

13. Gonzalez Gonzalez C, Sanchez Garcia S, Juarez Cedillo T, Rosas Carrasco O, Gutierrez Robledo L, Garcia Peńa C. Health care utilization in the elderly Mexican population: Expenditures and determinants. BMC Pub Health. 2011;11:192.

14. Puentes Rosas E, Gómez Dantés O, Garrido Latorre F. Trato a los usuarios en los Servicios Públicos de Salud en México. Rev Panam Salud Pública 2006;19(6):394-402.

15. Ortiz Espinosa RM, Muñoz Juárez S, Torres Carreño E. Satisfacción de los usuarios de 15 hospitales de Hidalgo, México. Rev Esp Salud Pública. 2004;78(4):527-37.

16. Medeiros FA, Araújo-Souza GC, Albuquerque-Barbosa AA, Clara-Costa IC. Aceptación en una Unidad Básica de Salud: enfoque en la satisfacción del usuario. Rev Salud Pública. 2010;12(3):402-13.

17. Costa MFBN, Ciosak SI. Comprehensive health care of the elderly in the Family Health Program: vision of health professionals. Rev Esc Enferm USP [Internet]. 2010 [cited 2011 Mar 23];44(2):437-44. Available from: http://www.scielo. br/pdf/reeusp/v44n2/en_28.pdf

18. Lee R, Mason A, Cotlear D. Some economic consequences of global aging [Internet]. 2010 [cited 2011 Mar 23]. Available from: http://siteresources.worldbank.org/HEALTHNUTRITIONANDPOPULATION/Resources/281627-1095698140167/SomeEconomicConsequencesOfGlobalAging.pdf

19. Coulter A. Can patients assess the quality of health care? BMJ. 2006;333(7557):1-2.

\section{Os autores agradecem ao Conselho Nacional de Ciência e Tecnologia (National Council on Science and Technology - CONACyT) pelo auxílio financeiro concedido a este projeto; à Dra. Hortensia Reyes e ao Dr. Javier Idrovo pela leitura criteriosa e sugestões feitas para a melhoria do manuscrito; a à Sra. Elizabeth Aguilar pelo apoio oferecido no trabalho de campo.}

